\title{
Annals of Nephrology
}

\section{Prophylaxis in kidney Transplantation}

\section{Massimo Gai*, Roberta Clari, Sergio Dellepiane, Davide Diena, Ana Manzione, Maria Messina and Luigi Biancone}

\author{
SCU Nephrology, Dialysis and Transplantation - AOU Città della Salute e della Scienza di Torino, University of \\ Torino, Italy
}

\begin{abstract}
Kidney transplant exposes patients to an increased risk of developing infections and tumors. Antiviral therapy and prophylaxis are one of the challenges of the modern transplant strategy. In recent years lots of progress has been made in preventing and managing CMV infection ensuring better outcomes and reducing CMV impact on morbidity, mortality and graft survival.
\end{abstract}

Keywords

Kidney transplantation, Viral infections, CMV infection, CMV prophylaxis, Ganciclovir, Valganciclovir

\section{Introduction}

Although kidney transplant is the therapy of choice for kidney failure, it exposes patients to an increased risk of developing infections and tumors due to immunosuppressive therapy.

Among these complications, cytomegalovirus (CMV) still remains one of the most important and frequent infections in renal transplant recipient with great impact on morbidity, mortality and graft survival since its various direct and indirect effects: increased risk for other bacterial, fungal and viral infections, and for posttransplant lymphoproliferaive disorders, graft dysfunction, acute and chronic rejection, histological changes, and vascular disease.

Human CMV is ubiquitous with a seroprevalence in the general population between $30 \%$ and $97 \%$, increasing with age; it can be transmitted through various ways such as human fluids (saliva, urine, blood transfusion), sexual contacts, placental transfer, breastfeeding, solid-organ transplantation or hematopoietic stem cell transplantation [1]

In kidney transplant CMV infection is related to many risk factors such as type and dosage of immunosoppressive drugs (for example cyclosporin and antilymphocyte antibodies both seem to increase the risk in contrast to basiliximab; sirolimus may have a protective effect; conflicting reports about mycophenolate mofetil), donor age (risk seems to increase with older donors), simultaneous kidney-pancreas transplantation, episodes of acute rejection and impaired graft function. The most important risk factor however seem to be the donor/recipient CMV serostatus: CMV seronegative recipients of $\mathrm{CMV}$ seronegative donors have the lowest incidence $(<5 \%)$ and infection may be caused by false seronegativity in one or both donor and recipient; CMV seronegative recipients of CMV seropositive donors are instead the class with the highest risk of primary infection which means the detection of CMV infection in seronegative patients or a seroconversion. The combinations donor seronegative/recipient seropositive or donor seropositive/recipient seropositive are considered as moderate risk; in the first case there can be only a reactivation of latent $\mathrm{CMV}$ virus due to immunosoppressive therapy; in the second case there can be the risk for both reactivation or superinfection with new viral strain [2-4].

It's important to distinguish between CMV infection and CMV disease. The first one is defined by the findings of: seroconversion (appearance of anti CMV immunoglobulin M antibodies), fourfold increase of pre-existing anti-CMV immunoglobulin $G$ titers, CMV antigens in infected cells, CMV DNAemia, isolation of the virus in cultures. CMV disease instead, may present with aspecific clinical signs and symptoms, such as fever or leukopenia, or with organ involvement (pneumonitis, hepatitis, pancreatitis, colitis, meningoencephalitis, chorioretinitis and myocarditis). Infection and disease can be both de novo or recurrent.

\section{Prophylactic Strategies}

Through the years two main approaches have been developed to prevent CMV disease in recipients of solid organ transplantation, the prophylactic strategy and the preemptive strategy. The first one consists in giving antiviral drugs to high risk patients starting in the immediate or very early post-transplant period and continuing

*Corresponding author: $\mathrm{Dr}$ Massimo Gai, SCU Nephrology, Dialysis and Transplantation - AOU Città della Salute e della Scienza di Torino, University of Torino, Corso Bramante 88, 10126 Torino, Italy, Tel: +39-0116335672, E-mail: massimogai@gmail.com

Received: November 22, 2016; Accepted: December 20, 2016; Published online: December 23, 2016

Citation: Gai M, Clari R, Dellepiane S, et al. (2016) CMV Prophylaxis in kidney Transplantation. Ann Nephrol 1(1):7-8

Copyright: @ 2016 Gai M, et al. This is an open-access article distributed under the terms of the Creative Commons Attribution License, which permits unrestricted use, distribution, and reproduction in any medium, provided the original author and source are credited. 
Citation: Gai M, Clari R, Dellepiane S, et al. (2016) CMV Prophylaxis in kidney Transplantation. Ann Nephrol $1(1): 7-8$

\begin{tabular}{|c|c|c|}
\hline & $\mathrm{CrCl}$ (ml/min) & Prophylactic dose \\
\hline \multirow{5}{*}{ Intravenous ganciclovir } & $\geq 70$ & $5 \mathrm{mg} / \mathrm{kg}$ once daily \\
\hline & $50-69$ & $2.5 \mathrm{mg} / \mathrm{kg}$ once daily \\
\hline & $25-49$ & $1.25 \mathrm{mg} / \mathrm{kg}$ once daily \\
\hline & $10-24$ & $0.625 \mathrm{mg} / \mathrm{kg}$ once daily \\
\hline & $<10$ & $0.635 \mathrm{mg} / \mathrm{kg} 3$ times a week - after hemodialysis \\
\hline \multirow{5}{*}{ Oral valganciclovir } & $\geq 70$ & 900 mg once daily \\
\hline & $50-69$ & $450 \mathrm{mg}$ once daily \\
\hline & $25-49$ & $450 \mathrm{mg}$ every 2 days \\
\hline & $10-24$ & 450 mg twice weekly \\
\hline & $<10$ & 100 mg 3 times a week - after hemodialysis \\
\hline
\end{tabular}

often up to 3 or 6 months; the second one consists instead in periodic monitoring for viremia to detect early viral replication and allow prompt treatment to prevent the progression to clinical disease; unfortunately there is a lack of consensus on the threshold level to be used to start treatment. Many trials compared the two strategies, but no significant differences were found between them in reducing the risk of CMV disease although the odds of developing late-onset infections (after the discontinuation of the therapy) as well as side effects, such as leukopenia and neutropenia, seemed to be higher among those receiving prophylaxis therapies [5]. Furthermore many centers use a hybrid strategy composed by both prophylaxis therapy and preemptive monitoring after completing the prophylaxis period.

Many researches are investigating a prophylactic vaccine to prevent cytomegalovirus infection [6]. An effective CMV vaccine would be beneficial in decreasing the need for anti-CMV drugs. CMV affects newborns and immunocompromised patients, making it a candidate for recommended universal vaccination. Several new CMV vaccine candidates are currently undergoing evaluation in clinical trials. Developing a vaccine to prevent CMV offers the best chance of substantially eliminating congenital CMV infection and drugs therapy in high risk patients $[7,8]$.

\section{Drugs and Prophylaxis}

Drugs used for prophylaxis, preemptive strategy and treatment of the disease are the same: the oldest one is the Ganciclovir which can now be administered only intravenously (the oral form is no longer available). However the most widely currently used drug is Valganciclovir: it is a prodrug of ganciclovir for oral administration and has a bioavailability of nearly $70 \%$ (bioavailability of oral ganciclovir is $7 \%$ instead); given at the doses of 450 or $900 \mathrm{mg}$ it guarantees serum ganciclovir levels equal to those obtained with intravenous ganciclovir at a dose of $2.5-5 \mathrm{mg} / \mathrm{kg}$. Doses of both drugs should be adjusted for kidney function to ensure efficacy and prevent toxicity (Table 1). Other antiviral agents are available: CMV hyperimmune globulin (human serum with high titer of anti-CMV antibodies); Valacyclovir which seems to be as effective as Valganciclovir in prophylaxis, but much more related to kidney damage and graft rejection; Leflunomide, currently used for the treatment of rheumatoid arthritis and BKV nephropathy.

Current international consensus guidelines suggest tailoring the prevention strategy on the different donor/recipient serostatus [4]:

- CMV-positive donor/CMV-negative recipient: a period of 6 months prophylaxis therapy is reccomended, especially when antilymphocyte antibodies are used for induction. Valganciclovir is now the drug of choice; there is no need for intravenous Ganciclovir;

- CMV-negative donor/CMV-positive recipient or CMV-positive donor/CMV-positive recipient: the use of prophylactic therapy (up to 3 months post-transplantation) should be evaluated especially in the cases of antilymphocyte antibodies induction or rejection treatment

- CMV-negative donor/CMV-negative recipient: based on the low risk of infection there is no recommendation for prophylaxis approach in these cases.

\section{Therapy}

When CMV disease develops international guidelines recommend the use of oral Valganciclovir, $900 \mathrm{mg}$ every $12 \mathrm{hr}$ in normal renal function (which is more convenient for both patient and caregiver, reduces hospitalization and avoids the risk of intravenous therapy) except in cases of life-threatening disease when optimal drug exposure is required (intravenous Ganciclovir preferred $5 \mathrm{mg} /$ $\mathrm{kg} \mathrm{q} 12 \mathrm{hr}$ in normal function) [4]. Duration of treatment depends on the monitoring of weekly CMV viral loads: therapy should be continued until one or two consecutive negative samples are obtained (minimum treatment course is two weeks).

After a successful treatment course a secondary prophylaxis, defined as prolonged therapy with standard prophylaxis doses from one to three months, can be given to the patient to reduce the risk of recurrent disease.

\section{Conclusion}

In conclusion, CMV infection is now one of the most important infections affecting recipients of solid organ transplant; however during the past years lots of progress has been made in preventing and managing this frequent complication ensuring better outcomes and reducing the CMV impact on morbidity, mortality and graft survival.

\section{References}

1. Beam E , Dioverti V, Razonable RR (2014) Emerging cytomegalovirus management strategies after solid organ transplantation: challenges and opportunities. Curr Infect Dis Rep 16: 419.

2. De Keyzer K, Van Laecke S, Peeters P, et al. (2011) Human cytomegalovirus and kidney transplantation: a clinician's update. Am J Kidney Dis 58: 118-126.

3. Kotton CN, Kumar D, Caliendo AM, et al. (2010) International consensus guidelines on the management of cytomegalovirus in solid organ transplantation. Transplantation 89: 779-795.

4. Kotton CN, Kumar D, Caliendo AM, et al. (2013) Updated international consensus guidelines on the management of cytomegalovirus in solid-organ transplantation. Transplantation 96: 333-360

5. Couzi L, Helou S, Bachelet T, et al. (2012) Preemptive therapy versus valgancyclovir prophylaxis in cytomegalovirus-positive kidney transplant recipients receiving antithymocyte globulin induction. Transplant Proc 44: 2809-2813.

6. Stratton KR, Durch JS, Lawrence RS (2000) Vaccines for the 21st Century: A Tool for Decisionmaking. The National Academies Collection: Reports funded by National Institutes of Health.

7. Boeckh M, Gilbert PB (2016) Search continues for a CMV vaccine for transplant recipients. Lancet Haematol 3: 58-59.

8. Plotkin SA (2002) Is there a formula for an effective CMV vaccine? J Clin Virol 2: S13-S21. 